\title{
A cross-sectional study of the association between persistent organic pollutants and glucose intolerance among Greenland Inuit
}

\author{
M. E. Jørgensen • K. Borch-Johnsen • P. Bjerregaard
}

Received: 27 December 2007 / Accepted: 8 May 2008/Published online: 17 June 2008

(C) Springer-Verlag 2008

\begin{abstract}
Aims/hypothesis Some evidence supports the hypothesis that persistent organic pollutants (POPs) may increase the risk of type 2 diabetes. The Inuit population in Greenland, which is highly exposed to POPs due to a high intake of marine mammals, has experienced a rapid increase in diabetes prevalence over the last 30 years. Thus the aim was to study the association between POPs and glucose intolerance and markers of insulin resistance and insulin secretion using a population-based design.

Methods From 1999 to 2002 the Greenland population study was carried out among adult Inuit living in Greenland. The examination included a 75 g OGTT, anthropometric measurements, a structured interview, and blood tests. Plasma glucose and serum insulin were analysed, and three defined subclasses of POPs were analysed in a subgroup. Associations were adjusted for age, sex, waist circumference, Inuit heritage, cigarette smoking, alcohol consumption and educational level.

Results Data on POPs were available on 692 individuals, 305 men (mean age 50 years) and 387 women (mean age 49 years). The prevalence of diabetes was $10.3 \%$, and $10.5 \%$ had impaired glucose tolerance. The concentrations of several POPs were exceptionally high. While no associations were found between POPs and stages of glucose intolerance or markers of insulin resistance, POPs were
\end{abstract}

M. E. Jørgensen $(\bowtie) \cdot K$. Borch-Johnsen

Steno Diabetes Center,

Gentofte, Denmark

e-mail: Mej@niph.dk

M. E. Jørgensen · P. Bjerregaard

Centre for Health Research in Greenland,

National Institute of Public Health,

Øster Farimagsgade 5A,

1399 Copenhagen, Denmark significantly inversely associated with stimulated insulin concentrations and homeostasis model assessment of beta cell function.

Conclusions/interpretation The study indicates that POPs may affect insulin secretion rather than being involved in the pathogenesis of insulin resistance. No association was seen between POPs and glucose intolerance or markers of insulin resistance.

Keywords Diabetes - Impaired glucose tolerance · Insulin resistance · Insulin secretion · Inuit .

Organochlorines $\cdot$ Pesticides

$\begin{array}{ll}\text { Abbreviations } \\ \text { AhR } & \text { aryl hydrocarbon receptor } \\ \text { DDE } & p, p^{\prime} \text {-dichlorodiphenyldichloroethene } \\ \text { DDT } & p, p^{\prime} \text {-dichlorodiphenyltrichloroethane } \\ \text { HCB } & \text { hexachlorobenzene } \\ \beta \text {-HCH } & \text { hexachlorocyclohexane } \\ \text { HOMA-B } & \text { homeostasis model assessment of beta cell } \\ & \text { function } \\ \text { HOMA-IR } & \text { homeostasis model assessment of insulin } \\ & \text { resistance } \\ \text { IGT } & \text { impaired glucose tolerance } \\ \text { LOD } & \text { limit of detection } \\ \text { OC } & \text { organochlorine } \\ \text { PCB } & \text { polychlorinated biphenyl } \\ \text { POP } & \text { persistent organic pollutant } \\ \text { TCDD } & 2,3,7,8 \text {-tetrachlorodibenzo- } p \text {-dioxin }\end{array}$

Introduction

Organic environmental contaminants refer to a large number of compounds found as contaminants in the 
environment as a consequence of industrial and agricultural pollution. A number of substances have been found to be persistent in the environment and are found as contaminants in foods today. Attention has been directed towards persistent organochlorine (OC) contaminants such as polychlorinated biphenyls (PCBs) and dioxins and compounds that were earlier widely used as pesticides (e.g. $p$, $p^{\prime}$-dichlorodiphenyltrichloroethane [DDT]). Exposure to dioxins has recently been associated with an increased risk of diabetes [1]. Prospective cohort studies of individuals exposed to 2,3,7,8-tetrachlorodibenzo- $p$-dioxin (TCDD), the most potent dioxin congener of persistent organic pollutants (POPs) in occupational or accidental settings, have reported increased risk of diabetes, modified glucose metabolism or insulin resistance [2-6]. Type 2 diabetes was associated with the exposure to dioxin-containing Agent Orange in Vietnam [7]. However, PCBs and pesticides have also been associated with diabetes in the Swedish Baltic Sea Fishermen study [8]. Two population-based studies have demonstrated a cross-sectional association between lowlevel exposure to some POPs and diabetes and insulin resistance; the NHANES study [9] and a Belgian study [10], and also longitudinal data from the Michigan PBB cohort showed that background exposure to PCBs was associated with incident diabetes after 25 years of follow-up [11].

OC compounds in humans may be derived from the pollution of the environment, where the compounds, being fat-soluble and because of their non-polar properties, accumulate up through the food chain. The Inuit population in Greenland is highly exposed to POPs due to a high intake of marine mammals. Concentrations of POPs among Inuit are amongst the highest in the world, and the age pattern indicates that bioaccumulation of PCBs started in the 1950 s $[12,13]$.

Over the past decades substantial changes have occurred in Greenland, with a rapid modernisation, especially in the towns, with a decline in consumption of traditional foods. Parallel to this, Greenland Inuit have experienced a rapid increase in diabetes prevalence [14]. Thus, the aim was in a population-based design to study the cross-sectional association between POPs and diabetes, impaired glucose regulation, markers of insulin resistance and insulin secretion in a highly exposed Inuit population.

\section{Methods}

Participants Data were collected from March 1999 to September 2002, in three selected areas in Greenland, representative of the population on the west coast of Greenland where $95 \%$ of the population live. The total population of Greenland is 56,000, of whom $90 \%$ are ethnic Greenlanders (Inuit). Genetically, Greenlanders are Inuit
(Eskimos) with an admixture of European genes. They are closely related to the Inuit and Yupik in Canada, Alaska and Siberia.

Until the 1950s most Greenlanders made their living by small-scale hunting and fishing. Hunting and fishing are still important leisure-time activities, and traditional marine food makes up approximately $20 \%$ of the diet.

The study sample was drawn from the central population register and consisted of Greenlanders aged $\geq 35$ years living in three areas of West Greenland, i.e. Nuuk (population 14,000), Qasigiannguit (population 1,400), and four villages in the district of Uummannaq (population 240-275 in each village). In Nuuk a random sample of the population was invited to participate, while in Qasigiannguit and Uummannaq all residents were invited. The details of this study have been described previously [15].

For all participants, informed consent was obtained in writing and orally. The study was approved by the Commission for Scientific Research in Greenland.

Physical measurements The participants underwent anthropometrical measurements, fasting blood samples, and a $75 \mathrm{~g}$ standardised OGTT. With the participant standing, waist circumference was measured midway between the iliac crest and the costal margin.

Participants with known diabetes did not have an OGTT, but fasting venous plasma glucose was measured. Plasma samples were immediately put on ice and centrifuged at $4^{\circ} \mathrm{C}$ within $30 \mathrm{~min}$ of sampling. Samples were stored frozen at $-20^{\circ} \mathrm{C}$, and shipped to Denmark for analyses. Plasma glucose was determined by the hexokinase/G6P-DH method (Boehringer, Mannheim, Germany). Diabetes was classified according to the WHO criteria 1999 [16]. Serum insulin was analysed using a fluoroimmunoassay technique (code no. K6219; Dako Diagnostics, Ely, UK) with a AutoDelfia measuring instrument (Perkin Elmer-Wallac, Finland) with an interassay precision $\mathrm{CV}<6 \%$. The laboratory of the Steno Diabetes Center, Denmark, performed the analyses of plasma glucose and serum insulin.

Homeostasis model assessment (HOMA) was used to calculate insulin resistance as described previously [17]. Basal insulin secretion was measured as HOMA of beta cell function (HOMA-B), which was calculated as (fasting plasma insulin $[\mathrm{pmol} / 1] \times 3.33) /($ fasting plasma glucose [mmol/1]-3.5).

Analyses of POPs were performed only in individuals with full food-frequency data. Analyses of POPs were performed at the laboratory of the Centre de Toxicologie du Québec, Canada, which is accredited by the Canadian Association for Environmental Analytical Laboratories. Blood samples containing EDTA as the anticoagulant were centrifuged and the plasma was collected in glass vials prewashed with hexane. Plasma samples were stored at 
$-20^{\circ} \mathrm{C}$ until analysis. A 1:1:3 mixture of ammonium sulphate:ethanol:hexane (wt/vol./vol.) was first added to the plasma to extract OCs. The extracts were then concentrated and purified on two Florisil columns (60100 mesh; Fisher Scientific, Nepean, ON, Canada). The 13 most prevalent PCB congeners (IUPAC nos 28, 52, 99, 101, 105, 118, 128, $138,153,163,156,170$ and 180) and 11 chlorinated pesticides or their metabolites (aldrin, mirex, $\alpha$-chlordane, $\gamma$ chlordane, DDT, $p, p^{\prime}$-dichlorodiphenyldichloroethene [DDE], hexachlorobenzene $[\mathrm{HCB}], \beta$-hexachlorocyclohexane $[\beta-\mathrm{HCH}]$, cis-nonachlor, trans-nonachlor and oxychlordane) were measured in the purified extracts with an HP 5890 high-resolution GC equipped with dual-capillary columns (HP Ultra I and Ultra II) and dual Ni-63 electron capture detectors (Hewlett-Packard, Palo Alto, CA, USA). Per cent recovery ranged from $89 \%$ to $100 \%$, and the detection limit was approximately $0.02 \mu \mathrm{g} / \mathrm{l}$ in the final extract for all compounds. CV values ( $n=20$, different days) ranged from 2.1 to $9.1 \%$. Biases (the difference between the concentration of the reference material and the concentration found using the analytic method) ranged from $10.9 \%$ to $3.8 \%$. POPs were reported on a standardised lipid-adjusted basis when relying on blood specimens for quantifying concentrations of lipophilic environmental contaminants [18]. Estimates of total serum lipids were calculated by summation of the individual lipid components by the formula: total plasma lipid $=1.677 \times($ total cholesterol $[\mathrm{g}]-$ non-esterified cholesterol $[\mathrm{g}])+$ non-esterified cholesterol (g)+triacylglycerol+ phospholipids (g). Lipids were measured using standard enzymatic procedures.

Three approaches to grouping POPs according to their properties were used to create exposure scales: dioxin-like PCBs, non-dioxin-like PCBs and OC pesticides.

Interview and questionnaire The survey questionnaires were developed in Danish and subsequently translated into Greenlandic. The translation procedure included translation by two or more interpreters followed by an independent back translation into Danish and revision of the translation as needed. Most information was obtained in the Greenlandic language. Participants were interviewed by trained interviewers as well as given a self-administered questionnaire.

Only Inuit defined as persons with at least one Inuit parent were included in the study.

Genetic heritage was estimated from questions on the ethnicity of the four grandparents, and if this information was missing, of the parents. It was subsequently recoded as full (all grandparents were Greenlanders) or partly Inuit heritage.

Participants were asked whether a physician had ever told them that they had diabetes. The frequency and amount of alcohol consumption was reported, and participants were classified as smokers (current smokers and past smokers) or non-smokers.

Individuals were asked about their physical activity levels within the last year. Physical activity was graded as (1) sedentary; (2) light; (3) physical activity $<4$ h per week; (4) physical activity at least $4 \mathrm{~h}$ per week; and (5) heavy activity several times per week. Because of small numbers in the groups it was recoded on a three-point scale: sedentary (1); moderate (2 and 3); and heavy physical activity (4 and 5). Education was recorded as the grade completed.

Statistics and data analysis Analyses were performed using SAS version 9.1 (SAS Institute, Carey, NC, USA). POPs for which at least $60 \%$ of the participants had concentrations above the limit of detection (LOD) were included. For samples below LOD, half of the detection limit was used as an estimate of the concentration. To yield a cumulative measure of the three defined subclasses of POPs we summed the ranks of each POP that belonged to the class, and the summary values were categorised by cut-off points of 25th, 50th and 75th percentile values. Analyses of POPs were performed lipid-adjusted and unadjusted in separate analyses, the latter to avoid a potential negative confounding of the association between POPs and glucose intolerance outcomes. The concentrations of contaminants, insulin and HOMA indices were not normally distributed. Consequently POPs were presented as median concentration and range. After natural logarithmic transformation, concentrations of contaminants, insulin and HOMA indices were normally distributed and the log-transformed values were used for the subsequent statistical analyses. Multiple logistic regression analyses were used to examine the association between quartiles of POPs and diabetes or impaired glucose tolerance (IGT), and general linear models were used to examine the association between quartiles of POPs and fasting and $2 \mathrm{~h}$ glucose and insulin and the association between POPs and HOMA-IR and HOMA-B for individuals without diabetes. Variables considered to be confounders in the multivariate analysis were age, sex, waist circumference, Inuit heritage, cigarette smoking, alcohol consumption and educational level.

\section{Results}

An OGTT was performed in 917 individuals aged $\geq 35$ years. Within this group, analyses of POPs were performed only in individuals with full food-frequency data. Thus the study population included in the present analyses comprised 692 Greenlanders. There was no difference between the groups with and without full food-frequency informa- 
tion with respect to distribution of sex and glucose tolerance status and with respect to mean age and waist circumference.

The participation rate was $67 \% ; 64 \%$ in Nuuk, $72 \%$ in Qasigiannguit, and 61\% in the villages in Uummannaq ( $p=$ 0.025 ). The mean age of the participants was 49 years, and $44 \%$ of the participants were men. The prevalence of diabetes was $10.3 \%, 11.2 \%$ among men and $9.7 \%$ among women; $10.5 \%$ met the criteria for IGT, $10.2 \%$ of men and $10.7 \%$ of women.

Table 1 shows median plasma concentrations of the three defined subclasses of POPs: three dioxin-like PCBs, ten non-dioxin-like $\mathrm{PCBs}$ and $11 \mathrm{OC}$ pesticides. Apart from aldrin, PCB52, PCB28, PCB128 and $\alpha$ - and $\gamma$-chlordane, compounds were detected in $>99 \%$ of the population. $\alpha$ Chlordane and $\gamma$-chlordane were only detected in $27 \%$ and $17 \%$ of the samples, with very low concentrations, whereas cis- and trans-nonachlor and oxychlordane were detected in all samples with median concentrations in the range of $100-500 \mu \mathrm{g} / \mathrm{g}$ lipid. PCB congeners with IUPAC numbers
138, 153 and 180 were present with the highest median concentrations.

Age and male sex were positively associated with all three subclasses of POPs. Waist circumference was positively associated with dioxin-like PCBs and OC pesticides. Full Inuit heritage was associated with higher concentrations of OC pesticides compared with mixed heritage. Those with low education had higher concentrations of all subclasses of POPs. Frequent alcohol consumption was positively associated with non-dioxin-like PCBs, while the association with $\mathrm{OC}$ pesticides and dioxin-like PCBs was negative. Current smoking was associated with higher concentrations of non-dioxin-like PCBs, whereas physical activity was not associated with POPs.

We constructed two logistic regression models with diabetes and IGT as dependent variables. Model 1 was adjusted for age and sex; model 2 was further adjusted for ethnicity, waist circumference, alcohol consumption, smoking and educational level (Table 2). There was no association between any of the defined subclasses of POPs

Table 1 Median concentrations and numbers below LOD of analysed POPs within quartiles of dioxin-like PCBs, non-dioxin-like PCBs and OC pesticides

\begin{tabular}{|c|c|c|c|c|c|c|}
\hline \multirow[t]{2}{*}{ Parameter } & \multirow[t]{2}{*}{ Below LOD $(n)$} & \multicolumn{5}{|c|}{ Median (ng/g lipid) in quartiles } \\
\hline & & $1 \mathrm{st}$ & 2 nd & $3 \mathrm{rd}$ & 4 th & Total range \\
\hline \multicolumn{7}{|l|}{ Dioxin-like PCBs } \\
\hline PCB105 & 0 & 6.5 & 15 & 25 & 44 & $1.5-160$ \\
\hline PCB118 & 0 & 43 & 99 & 170 & 300 & $4.4-860$ \\
\hline PCB156 & 0 & 20 & 46 & 70 & 130 & $2.3-510$ \\
\hline \multicolumn{7}{|c|}{ Non-dioxin-like PCBs } \\
\hline PCB28 & 272 & 4.9 & 5.5 & 6.5 & 8.5 & $0.38-46$ \\
\hline PCB52 & 624 & 7.2 & 8.9 & 13 & 14 & $2.3-88$ \\
\hline PCB99 & 0 & 35 & 74 & 120 & 190 & $2.8-590$ \\
\hline PCB101 & 0 & 4.6 & 8.3 & 12 & 17 & $0.94-67$ \\
\hline PCB128 & 33 & 1.6 & 3.2 & 4.4 & 6.5 & $0.16-28$ \\
\hline PCB138 & 0 & 130 & 280 & 440 & 730 & $17-1900$ \\
\hline PCB153 & 0 & 290 & 620 & 995 & 1,700 & $38-4300$ \\
\hline PCB163 & 0 & 54 & 130 & 220 & 390 & $4.9-940$ \\
\hline PCB170 & 0 & 46 & 100 & 170 & 310 & $7.6-1100$ \\
\hline PCB180 & 0 & 150 & 350 & 580 & 1,100 & $24-4200$ \\
\hline \multicolumn{7}{|l|}{ OC pesticides } \\
\hline Aldrin & 685 & - & - & 0.44 & 0.7 & $0.4-0.71$ \\
\hline Mirex & 0 & 7.9 & 23 & 42 & 83 & $0.44-380$ \\
\hline $\mathrm{HCB}$ & 0 & 120 & 280 & 460 & 770 & 23-1900 \\
\hline$\beta-\mathrm{HCH}$ & 0 & 15 & 29 & 43 & 66 & $1.6-180$ \\
\hline$\alpha$-Chlordane & 434 & 0.31 & 0.50 & 0.62 & 1.20 & $0.12-5.4$ \\
\hline$\gamma$-Chlordane & 575 & 0.15 & 0.19 & 0.51 & 1.15 & $0.13-6.6$ \\
\hline Oxychlordane & 0 & 54 & 170 & 345 & 620 & $1.5-2200$ \\
\hline trans-Nonachlor & 1 & 120 & 350 & 700 & 1,200 & $0.8-3400$ \\
\hline cis-Nonachlor & 0 & 23 & 61 & 110 & 190 & $0.7-550$ \\
\hline DDT & 0 & 12 & 24 & 38 & 67 & $2.6-270$ \\
\hline DDE & 0 & 490 & 1,100 & 1,900 & 3,400 & $52-9200$ \\
\hline
\end{tabular}


and diabetes or IGT. Further adjustment for confounders did not change this finding.

We constructed general linear regression models with fasting and $2 \mathrm{~h}$ glucose and insulin and HOMA-IR and HOMA-B as dependent variables and quartiles of concentrations of POP subclasses as independent variables. Participants with diabetes were excluded from these analyses. There was an initial unadjusted association

Table 2 ORs and 95\% CIs of prevalence of diabetes and IGT by quartiles of dioxin-like PCBs, non-dioxin-like PCBs and $\mathrm{OC}$ pesticides according to demographic and behavioural characteristics $(n=692)$

\begin{tabular}{|c|c|c|}
\hline Parameter & Model 1 & Model 2 \\
\hline \multicolumn{3}{|c|}{ Diabetes vs non-diabetes } \\
\hline \multicolumn{3}{|c|}{ Dioxin-like PCBs } \\
\hline $1 \mathrm{st}$ & Reference & Reference \\
\hline $2 \mathrm{nd}$ & $1.3(0.4-3.3)$ & $1.6(0.6-4.1)$ \\
\hline $3 \mathrm{rd}$ & $2.1(0.9-5.2)$ & $1.9(0.74-5.1)$ \\
\hline 4th & $1.4(0.5-3.8)$ & $1.2(0.4-3.6)$ \\
\hline$p$ value & 0.28 & 0.37 \\
\hline \multicolumn{3}{|c|}{ Non-dioxin-like PCBs } \\
\hline $1 \mathrm{st}$ & Reference & Reference \\
\hline 2nd & $0.7(0.3-1.6)$ & $0.7(0.3-1.6)$ \\
\hline $3 \mathrm{rd}$ & $1.1(0.5-2.4)$ & $1.1(0.4-2.8)$ \\
\hline 4th & $1.0(0.4-2.4)$ & $1.2(0.4-3.2)$ \\
\hline$p$ value & 0.62 & 0.42 \\
\hline \multicolumn{3}{|c|}{ OC pesticides } \\
\hline $1 \mathrm{st}$ & Reference & Reference \\
\hline 2 nd & $1.7(0.7-4.5)$ & $1.9(0.7-5.1)$ \\
\hline $3 \mathrm{rd}$ & $1.3(0.4-3.6)$ & $1.2(0.4-3.6)$ \\
\hline 4th & $1.8(0.7-5.1)$ & $1.8(0.6-5.5)$ \\
\hline$p$ value & 0.45 & 0.4 \\
\hline \multicolumn{3}{|c|}{ IGT vs non-IGT } \\
\hline \multicolumn{3}{|c|}{ Dioxin-like PCBs } \\
\hline $1 \mathrm{st}$ & Reference & Reference \\
\hline 2 nd & $0.6(0.3-1.5)$ & $0.8(0.3-1.9)$ \\
\hline $3 \mathrm{rd}$ & $0.9(0.4-2.0)$ & $0.9(0.3-2.2)$ \\
\hline 4th & $0.6(0.3-1.6)$ & $0.7(0.2-1.8)$ \\
\hline$p$ value & 0.66 & 0.82 \\
\hline \multicolumn{3}{|c|}{ Non-dioxin-like PCBs } \\
\hline $1 \mathrm{st}$ & Reference & Reference \\
\hline 2nd & $0.6(0.3-1.4)$ & $0.7(0.3-1.6)$ \\
\hline $3 \mathrm{rd}$ & $0.7(0.3-1.6)$ & $0.8(0.3-2.0)$ \\
\hline 4 th & $0.3(0.1-0.9)$ & $0.5(0.2-1.3)$ \\
\hline$p$ value & 0.10 & 0.4 \\
\hline \multicolumn{3}{|c|}{ OC pesticides } \\
\hline $1 \mathrm{st}$ & Reference & Reference \\
\hline 2nd & $0.9(0.4-2.1)$ & $1.0(0.4-2.4)$ \\
\hline $3 \mathrm{rd}$ & $0.9(0.4-2.2)$ & $1.0(0.4-2.7)$ \\
\hline 4 th & $0.6(0.2-1.6)$ & $0.6(0.2-1.8)$ \\
\hline$p$ value & 0.63 & 0.55 \\
\hline
\end{tabular}

Model 1: adjusted for age and sex

Model 2: adjusted for age, sex, ethnicity, waist circumference, physical activity, alcohol consumption, smoking and educational level between dioxin-like and non-dioxin-like PCBs and fasting and $2 \mathrm{~h}$ glucose and insulin. The means of fasting and $2 \mathrm{~h}$ glucose and insulin adjusted for age, sex, ethnicity, waist circumference, physical activity, alcohol consumption, smoking and educational level are shown in Table 3. For all subclasses of POPs we found a significant inverse association between increasing concentrations of POPs and $2 \mathrm{~h}$ insulin, whereas no association was seen between POPs and glucose and fasting insulin, respectively.

While there was no association between any of the POP subclasses with HOMA-IR (data not shown), concentrations of dioxin-like PCBs and non-dioxin-like PCBs were significantly inversely associated with HOMA-B with a decrease in HOMA-B of $8.5 \%$ to $14.2 \%$ for increasing quartiles of dioxin-like PCBs and non-dioxin-like PCBs.

We repeated the analyses for POP-levels unadjusted for lipids and found the same associations between POPs and diabetes, IGT, $2 \mathrm{~h}$ glucose, insulin and HOMA indices (data not shown). Only for fasting glucose was a significant positive association with non-dioxin-like PCBs found.

We included an interaction term to test if sex or Inuit heritage statistically significantly modified the association between POPs and glucose tolerance status ( $p<0.05$ for the interaction term). This proved not to be the case; thus analyses were run only for the combined groups.

\section{Discussion}

The concentrations of PCBs and chlorinated pesticides were high among Greenland Inuit. Concentrations of PCBs and chlordanes were 10-12 times higher than in the NHANES study [9]; DDE concentration was twice as high. PCB153 was five times higher than in the Swedish Baltic Sea Fishermen study [8], DDE was twice as high. However, comparisons with other studies must be made carefully because of differences in methods of analysis and differences in age distribution.

We found no association between POPs and diabetes, IGT or markers of insulin resistance. This is consistent with findings of no association in other studies of highly exposed individuals, whereas a positive association is predominantly found in populations with only low-level background exposure [19]. One potential reason why our study does not confirm the finding of an association between POPs and glucose intolerance could be the existence of a threshold for POP concentration. The very high levels of contaminants and the high prevalence of diabetes among Greenland Inuit could indicate that most of the population is significantly exposed, and that levels above such a threshold do not add further risk.

Alternatively, metabolic disturbances related to insulin resistance and diabetes might reduce clearance and influ- 
Table 3 Mean concentrations and 95\% CIs for fasting and $2 \mathrm{~h}$ glucose and insulin according to quartiles of dioxin-like PCBs, non-dioxin-like PCBs and OC pesticides for participants without diabetes $(n=621)$

\begin{tabular}{|c|c|c|c|c|}
\hline Parameter & Mean fasting glucose $(\mathrm{mmol} / \mathrm{l})$ & Mean $2 \mathrm{~h}$ glucose $(\mathrm{mmol} / \mathrm{l})$ & Mean $^{\mathrm{a}}$ fasting insulin $(\mathrm{pmol} / \mathrm{l})$ & Mean $^{\mathrm{a}} 2 \mathrm{~h}$ insulin $(\mathrm{pmol} / \mathrm{l})$ \\
\hline \multicolumn{5}{|c|}{ Dioxin-like PCBs } \\
\hline $1 \mathrm{st}$ & $5.1(4.9-5.4)$ & $5.6(4.9-6.3)$ & $42(35-52)$ & $129(89-186)$ \\
\hline 2nd & $5.2(4.9-5.4)$ & $5.7(5.0-6.4)$ & $39(32-48)$ & $109(77-156)$ \\
\hline $3 \mathrm{rd}$ & $5.2(4.9-5.4)$ & $5.6(4.9-6.3)$ & $38(32-47)$ & $103(73-147)$ \\
\hline 4th & $5.385 .0-5.6)$ & $5.5(4.8-6.2)$ & $37(30-45)$ & $90(62-130)$ \\
\hline$p$ value & 0.24 & 0.68 & 0.77 & 0.04 \\
\hline \multicolumn{5}{|c|}{ Non-dioxin-like PCBs } \\
\hline $1 \mathrm{st}$ & $5.2(4.9-5.4)$ & $5.7(5.0-6.4)$ & $42(35-52)$ & $122(85-175)$ \\
\hline 2nd & $5.2(4.9-5.5)$ & $5.6(5.0-6.3)$ & $40(33-49)$ & $116(65-134)$ \\
\hline $3 \mathrm{rd}$ & $5.2(4.9-5.4)$ & $5.6(4.9-6.3)$ & $40(33-48)$ & $94(65-134)$ \\
\hline 4 th & $5.3(5.0-5.6)$ & $5.4(4.7-6.1)$ & $37(30-46)$ & $90(62-131)$ \\
\hline$p$ value & 0.25 & 0.75 & 0.37 & 0.01 \\
\hline \multicolumn{5}{|c|}{ OC pesticides } \\
\hline $1 \mathrm{st}$ & $5.2(4.9-5.4)$ & $5.6(4.9-6.3)$ & $41(33-50)$ & $133(92-192)$ \\
\hline $2 \mathrm{nd}$ & $5.2(4.9-5.4)$ & $5.7(5.0-6.4)$ & $39(32-48)$ & $114(80-164)$ \\
\hline $3 \mathrm{rd}$ & $5.2(5.0-5.6)$ & $5.6(4.8-6.2)$ & $39(32-47)$ & $101(71-145)$ \\
\hline 4th & $5.1(4.9-5.4)$ & $5.6(4.8-6.3)$ & $40(33-50)$ & $87(60-125)$ \\
\hline$p$ value & 0.38 & 0.74 & 0.15 & 0.03 \\
\hline
\end{tabular}

${ }^{\text {a }}$ Geometric mean

ence distribution of lipophilic compounds such as POPs. If a reverse causality exists, serum concentrations of POPs in the low-level range are proxy markers of dysmetabolism rather than biologically associated with diabetes and insulin resistance [19]. Lipid adjustment of POP concentrations to some extent compensates for this, although lipid standardisation has also been reported to be prone to bias [20]. Inclusion of POPs unadjusted for lipids in the analyses did not, however, change our findings.

POPs are suggested to influence insulin sensitivity by a decrease in the expression of the insulin-responsive glucose transporter GLUT4 [21], and by interaction with peroxisome proliferator-activated receptor- $\gamma$, a ligand-activated transcription factor linked with type 2 diabetes [1].

The toxicity of POPs is mediated partly through binding to the aryl hydrocarbon receptor (AhR), which is an intracellular ligand-dependent transcriptional factor expressed in most tissues of mammals. A study of men in Greenland, Sweden, Poland and Ukraine found significantly lower median levels of AhR TCDD toxic equivalents among the Inuit compared with the European groups [22], irrespective of serum concentrations of contaminants. The reason for these ethnic differences is unknown. Thus, low AhR-associated xenobiotic activity of POPs despite very high serum concentrations may contribute to the explanation of why we find no association between POPs and glucose intolerance among Inuit. More studies in this area are needed to clarify whether measurements of AhR activity levels in future studies could add information about potential health effects of POPs.
Another explanation for why we do not find an association between POPs and diabetes or insulin resistance could be that a potential negative health effect of contaminants is counterbalanced by beneficial dietary factors. Foods account for almost all exposure to POPs in Greenland, and especially $n-3$ fatty acids from fish and marine mammals are suggested to affect insulin sensitivity by changing the fatty acid composition of membrane lipids. Studies have shown that a high proportion of $n-3$ fatty acids may improve insulin signalling by increasing membrane fluidity [23, 24].

While no association was found between POPs and stages of glucose intolerance or with insulin resistance markers, there was, nevertheless, a significant negative association between all three subclasses of POPs and indices of insulin secretion: stimulated insulin and HOMA-B. Thus, our data indicate that POPs may affect basal and stimulated insulin secretion in non-diabetic individuals rather than being involved in the pathogenesis of insulin resistance.

One study has shown an association between PCBs and type 1 diabetes among pregnant women [25], and a high prevalence of GAD antibodies was found in a study of factory workers exposed to PDBs, supporting an immunomodulatory effect of PCBs [26]. Furthermore, animal studies have demonstrated a potential mechanism for PCB-related insulin release [27].

There were some inherent limitations in our study. Null findings always question whether the study had sufficient statistical power to show an association. However, after simple adjustment for age and sex the size of the ORs and 
the mean values of glucose and insulin did not indicate a clinically important association that a larger study would be able to detect. CV values and biases for POPs were comparable with other studies, although high for some POPs, which may potentially weaken an association between POPs and glucose intolerance.

The strength in our study relies on the diagnosis of diabetes and IGT, which were based on a glucose tolerance test. Only $30 \%$ of the participants with diabetes were aware of their diabetic status. In most other studies addressing POPs and diabetes, the diagnosis of diabetes is based on self-reported diabetes and the possibility exists that individuals with diabetes have changed their diet towards consuming more fatty fish according to dietary recommendations. Thus, change in dietary habits after the diagnosis of diabetes is a potential positive confounder.

In conclusion, we found that POPs were inversely associated with stimulated insulin concentrations and HOMA-B in non-diabetic individuals. Our findings, however, cannot confirm an association between POPs and stages of glucose intolerance or markers of insulin resistance in a highly exposed Inuit population. Further epidemiological and experimental studies of the role of POPs in insulin secretion are warranted.

Acknowledgements The Greenland population study was funded by the Danish Medical Research Council, the Greenland Medical Research Council, the Commission for Scientific Research in Greenland, the Danish Diabetes Association, the Health Insurance Foundation, the Emil Hertz Foundation and the Karen Elise Jensen's Foundation.

Conflict of interest The authors declare that there is no duality of interest associated with this manuscript.

\section{References}

1. Remillard RB, Bunce NJ (2002) Linking dioxins to diabetes: epidemiology and biologic plausibility. Environ Health Perspect 110:853-858

2. Steenland K, Calvert G, Ketchum N, Michalek J (2001) Dioxin and diabetes mellitus: an analysis of the combined NIOSH and Ranch Hand data. Occup Environ Med 58:641-648

3. Bertazzi PA, Bernucci I, Brambilla G, Consonni D, Pesatori AC (1998) The Seveso studies on early and long-term effects of dioxin exposure: a review. Environ Health Perspect 106(Suppl 2):625-633

4. Vena J, Boffetta P, Becher H et al (1998) Exposure to dioxin and nonneoplastic mortality in the expanded IARC international cohort study of phenoxy herbicide and chlorophenol production workers and sprayers. Environ Health Perspect 106(Suppl 2):645-653

5. Steenland K, Piacitelli L, Deddens J, Fingerhut M, Chang LI (1999) Cancer, heart disease, and diabetes in workers exposed to 2,3,7,8tetrachlorodibenzo-p-dioxin. J Natl Cancer Inst 91:779-786

6. Longnecker MP, Michalek JE (2000) Serum dioxin level in relation to diabetes mellitus among Air Force veterans with background levels of exposure. Epidemiology 11:44-48

7. Henriksen GL, Ketchum NS, Michalek JE, Swaby JA (1997) Serum dioxin and diabetes mellitus in veterans of Operation Ranch Hand. Epidemiology 8:252-258
8. Rylander L, Rignell-Hydbom A, Hagmar L (2005) A crosssectional study of the association between persistent organochlorine pollutants and diabetes. Environ Health 4:28

9. Lee DH, Lee IK, Song KE et al (2006) A strong dose-response relation between serum concentrations of persistent organic pollutants and diabetes: results from the National Health and Examination Survey. Diabetes Care 29:1638-1644

10. Fierens S, Mairesse H, Heilier JF et al (2003) Dioxin/polychlorinated biphenyl body burden, diabetes and endometriosis: findings in a population-based study in Belgium. Biomarkers 8:529-534

11. Vasiliu O, Cameron L, Gardiner J, Deguire P, Karmaus W (2006) Polybrominated biphenyls, polychlorinated biphenyls, body weight, and incidence of adult-onset diabetes mellitus. Epidemiology 17:352-359

12. Bjerregaard P, Dewailly E, Ayotte P, Pars T, Ferron L, Mulvad G (2001) Exposure of Inuit in Greenland to organochlorines through the marine diet. J Toxicol Environ Health A 62:69-81

13. AMAP (2004) AMAP assessment 2002: persistent organic pollutants in the Arctic. Arctic Monitoring and Assessment Programme (AMAP), Oslo, Norway

14. Jørgensen ME, Bjerregaard P, Borch-Johnsen K (2002) Diabetes and impaired glucose tolerance among the Inuit population of Greenland. Diabetes Care 25:1766-1771

15. Bjerregaard P, Curtis T, Borch-Johnsen K et al (2003) Inuit Health in Greenland: A population survey of life style and disease in Greenland and among Inuit living in Denmark. Int J Circumpolar Health 62(Suppl 1):1-79

16. WHO (1999) Definition, diagnosis and classification of diabetes mellitus and its complications. Part 1: diagnosis and classification of diabetes mellitus. Report of a WHO consultation. WHO/NCD/ NCS/99.2. WHO, Geneva

17. Matthews DR, Hosker JP, Rudenski AS, Naylor BA, Treacher DF, Turner RC (1985) Homeostasis model assessment: insulin resistance and beta-cell function from fasting plasma glucose and insulin concentrations in man. Diabetologia 28:412-419

18. Phillips DL, Pirkle JL, Burse VW, Bernet JT, Henderson LO, Needham LL (1989) Chlorinated hydrocarbon levels in human serum: effects of fasting and feeding. Arch Environ Contam Toxicol 18:495-500

19. Longnecker MP (2006) Pharmacokinetic variability and the miracle of modern analytical chemistry. Epidemiology 17:350-351

20. Schisterman EF, Whitcomb BW, Buck-Louis GM, Louis TA (2005) Lipid adjustment in the analysis of environmental contaminants and human health risks. Environ Health Perspect 113:853-857

21. Fujiyoshi PT, Michalek JE, Matsumura F (2006) Molecular epidemiologic evidence for diabetogenic effects of dioxin exposure in U.S. Air force veterans of the Vietnam war. Environ Health Perspect 114:1677-1683

22. Long M, Andersen BS, Lindh CH et al (2006) Dioxin-like activities in serum across European and Inuit populations. Environ Health 5:14

23. Salmerón J, Hu FB, Manson JE et al (2001) Dietary fat intake and risk of type 2 diabetes in women. Am J Clin Nutr 73:1019-1026

24. Storlien LH, Baur LA, Kriketos AD et al (1996) Dietary fats and insulin action. Diabetologia 39:621-631

25. Longnecker MP, Klebanoff MA, Brock JW, Zhou H, Collaborative Perinatal Project (CPP) (2001) Polychlorinated biphenyl serum levels in pregnant subjects with diabetes. Diabetes Care 24:1099-1101

26. Langer P, Tajtáková M, Guretzki HJ et al (2002) High prevalence of anti-glutamic acid decarboxylase (anti-GAD) antibodies in employees at a polychlorinated biphenyl production factory. Arch Environ Health 57:412-415

27. Fischer LJ, Wagner MA, Madhukar BV (1999) Potential involvement of calcium, CaM kinase II, and MAP kinases in PCB-stimulated insulin release from RINm5F cells. Toxicol Appl Pharmacol 159:194-203 